\title{
Estudio Morfológico y Biométrico de los Vínculos de los Tendones de los Músculos Flexores Superficial y Profundo de los Dedos de la Mano Humana
}

\author{
Anatomic and Biometric Study of the Vincula of the Tendons of Flexor \\ Digitorum Superficialis and Profundus Muscles of the Human Hand
}

\author{
E. Pereira*; S. A. Garbelotti"; V. Rodrigues Pereira*; R. Rotter Montibeller*; N. Wafae* \& E. Olave**
}

PEREIRA, E.; GARBElOTTI, S. A.; PEREIRA, V. R.; MONTIBELLER, R. R.; WAFAE, N. \& OLAVE, E. Estudio morfológico y biométrico de los vínculos de los tendones de los músculos flexores superficial y profundo de los dedos de la mano humana. Int. $J$. Morphol., 34(2):679-683, 2016.

RESUMEN: Los vínculos son estructuras especializadas inherentes a los tendones flexores de los dedos, y tienen gran importancia en la nutrición de los mismos, además de facilitar la flexión de los dedos después de una transección distal del tendón (es), permitiendo que éste (os) actuen indirectamente a través de las articulaciones interfalángicas. El presente estudio tuvo como objetivo revisar aspectos morfofuncionales de los vínculos largos y cortos de los músculos flexores de los dedos de la mano. El trabajo fue realizado en 60 manos de cadáveres formolizados de individuos adultos, brasileños, con una edad promedio de 53,1 $\pm 17,8$ años. Los vínculos cortos fueron encontrados en $100 \%$ de los casos, tanto en los músculos flexores superficiales de los dedos como en los profundos, siempre en número de uno en cada tendón y su localización siempre estuvo en la extremidad del tendón, antes de la inserción de éste en la articulación interfalángica proximal. Los vínculos largos presentaron gran variación morfológica, con una presencia promedio de $61,3 \%$ en los tendones del músculo flexor superficial y de 77,9 \% en el músculo flexor profundo. El número de vínculos por tendón varió aún más, no siendo posible identificar un patrón independientemente del músculo. En relación a la localización de los vínculos largos, en los tendones del músculo flexor superficial de los dedos, están insertos en la vaina fibrosa de la falange proximal, con un trayecto oblicuo y próximo a la articulación interfalángica proximal; los vínculos de los tendones del músculo flexor profundo estaban localizados en su mayoría $(86,7 \%$ ) entre los tendones a nivel de la articulación interfalángica proximal, presentando un trayecto rectilíneo entre esos dos tendones, mientras que en 13,3\% estuvieron localizados muy próximos a la abertura del tendón del músculo flexor superficial para el paso del tendón del músculo flexor profundo. Los vínculos son estructuras escenciales en la mantención de la nutrición y de los movimientos de los dedos aún en presencia de lesiones, por lo tanto, es importante tener una comprensión clara de ellos para una correcta evaluación clínica pensando en la reparación, reconstrucción y rehabilitación de las disfunciones de la mano.

PALABRAS CLAVE: Mano; Músculos flexores de los dedos; Vínculos largos y cortos.

\section{INTRODUCCIÓN}

La complejidad de la función de la mano es un reflejo de la anatomía de sus estructuras. Entre éstas, los músculos flexores tienen una arquitectura relativamente consistente, aunque presentan variaciones anatómicas notables a nivel de sus tendones. Los movimientos de los dedos son el resultado de un delicado equilibrio de los músculos flexores y extensores, que otorgan una increíble versatilidad de funciones, las que son fácilmente afectadas por traumas o enfermedades. Por ser ampliamente utilizada en las actividades cotidianas, deportivas u ocupacionales, la mano es una de las partes del cuerpo más frecuentemente lesionada. Comprender su compleja anatomía es de gran importancia para evaluar, reparar, reconstruir y rehabilitar sus funciones (Von Schroeder \& Botte, 1997).

Los vínculos son estructuras especializadas provenientes del mesotendón e inherentes a los tendones de los músculos flexores de los dedos, teniendo gran importancia para su nutrición (Shao, 1995), por lo que su conocimiento se torna fundamental en casos de laceración de los tendones, pues permite la comprensión de los mecanismaos de la respuesta biológica involucrados en la cicatrización tisular (Amadio et al., 1985). Además, los vínculos pueden ser importantes mecánicamente pudiendo facilitar la flexión de los

\footnotetext{
* Disciplina de Anatomia Descritiva e Topográfica, Universidade Federal de Sao Paulo, São Paulo, Brasil.

${ }^{*}$ Facultad de Medicina, Universidad de La Frontera, Temuco, Chile.
} 
dedos después de una transección distal de tendones, permitiendo a éstos actuar a través de las articulaciones interfalángicas (Stewart et al., 2007).

Debido a la importancia de las estructuras mencionadas, el presente trabajo tuvo como objetivo describir los vínculos cortos y largos en los tendones de los músculos flexores superficial y profundo de los dedos de la mano, su localización, número y registros biométricos tales como longitud y anchura, características que serán un aporte al conocimiento anatómico y anatomoclínico de la mano humana.

\section{MATERIAL Y MÉTODO}

Para este estudio observacional descriptivo, se utilizaron 60 manos de cadáveres formolizados de individuos adultos, Brasileños, sexo masculino y de edades entre 26 y 79 años, provenientes del Laboratorio de la Disciplina de Anatomía Descriptiva y Topográfica de la Universidade Federal de Sao Paulo - Escola Paulista de Medicina (UNIFESP-EPM).

Por tratarse de un estudio descriptivo y no comparativo, se aplicó un tratamiento estadístico inferencial, con valores absolutos, porcentaje de incidencia, promedio y desviación standard de los datos biométricos.

\section{RESULTADOS}

Vínculos cortos (Fig. 1). Estuvieron presentes en los tendones de ambos músculos flexores de los dedos, superficial y profundo, en $100 \%$ de los casos. El número de vínculos siempre fue uno en todos los dedos y su localización siempre estuvo en la extremidad del tendón, antes de la inserción de éste en la articulación interfalángica proximal. Presenta una forma de arco, de doble convexidad, semejante a un abanico; su parte anterior se inserta en la cara posterior del tendón y su parte posterior se fija en la vaina fibrosa, en el tercio distal de la falange proximal.

Vínculos largos. Los vínculos largos son más frecuentes en los dedos indicador y medio, en los tendones de ambos músculos flexores, siendo más común que se presente un vínculo por tendón, aunque puede haber dos o tres vínculos con cierta frecuencia en el dedo indicador. La frecuencia de los vínculos largos en valores absolutos y porcentuales por dedo se muestra en la Tabla I. En los tendones del músculo flexor superficial de los dedos, estos vínculos se encontraban localizados entre el tendón y la falange proximal, con

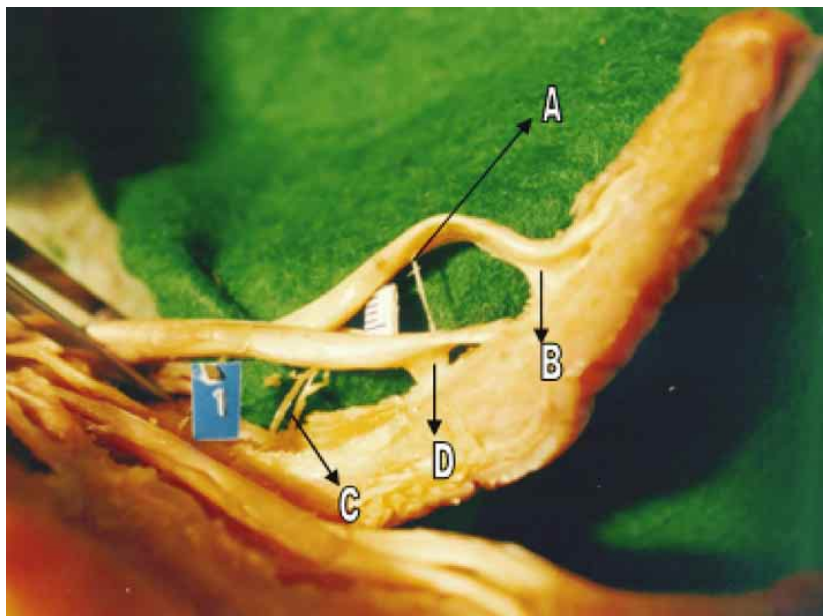

Fig. 1. Vínculos largos y cortos en el dedo medio de una mano derecha. $A=$ Vínculo largo en el tendón del músculo flexor profundo de los dedos; $\mathrm{B}=$ Vínculo corto en el tendón del músculo flexor profundo de los dedos; $\mathrm{C}=$ Vínculo largo (doble) en el tendón del músculo flexor superficial de los dedos y $\mathrm{D}=$ Vínculo corto en el tendón del músculo flexor superficial de los dedos.

Tabla I. Frecuencia de los vínculos largos en los tendones de los músculos flexores de los dedos (MFSD y MFPD).

\begin{tabular}{lcccc}
\hline & \multicolumn{2}{c}{ MFSD } & \multicolumn{2}{c}{ MFPD } \\
\cline { 2 - 5 } Dedos & $\boldsymbol{n ( 6 0 )}$ & $\mathbf{\%}$ & $\boldsymbol{n ~ ( 6 0 )}$ & $\mathbf{\%}$ \\
\hline Indicador & 40 & 66,7 & 53 & 88,2 \\
Medio & 44 & 73,3 & 46 & 76,7 \\
Anular & 35 & 58,3 & 45 & 75 \\
Mínimo & 28 & 46,7 & 43 & 71,2 \\
\hline
\end{tabular}

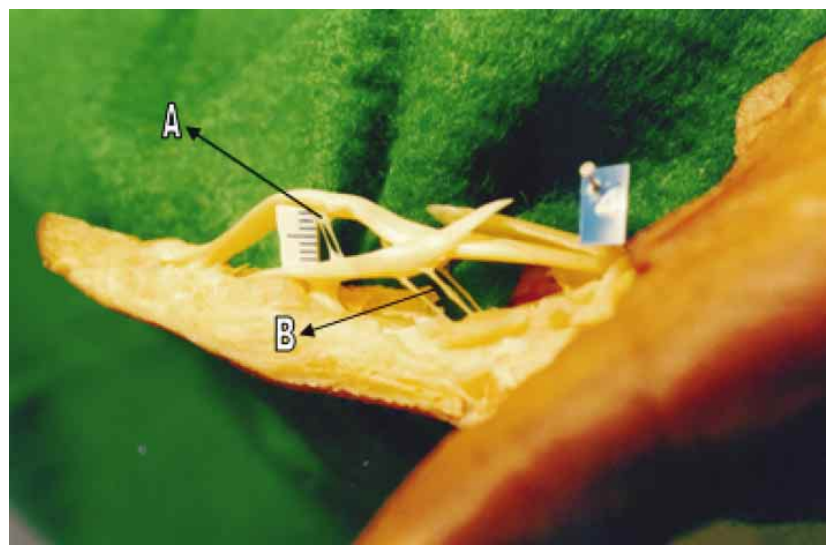

Fig. 2. Vínculos largos múltiples en el dedo indicador de una mano izquierda. A= Vínculo largo (dos) en el tendón del músculo flexor profundo de los dedos y $\mathrm{B}=$ Vínculo largo (tres) en el tendón del músculo flexor superficial de los dedos.

fijación en el tendón próximo a la articulación interfalángica proximal en $100 \%$ de los casos. En la Figura 2 se observan, los vínculos de dirección oblicua, insertos tanto en el tendón como en la vaina fibrosa. 
La mayor parte de los vínculos largos $(81,2 \%)$ estuvieron localizados a nivel de la articulación interfalángica proximal, entre los tendones de los músculos flexores superficial y profundo, presentando un trayecto rectilíneo entre esos dos tendones; en 8 casos $(13,3 \%)$ se localizaron próximos a la abertura del tendón del músculo flexor superficial de los dedos para el paso del tendón del flexor profundo (Fig. 2); en 2 casos (3,3\%) se presentaron paralelamente al tendón del flexor profundo de los dedos, al lado de la abertura del tendón del flexor superficial y en un caso (1,67 $\%)$ se observó un vínculo doble en forma de "V". Cuando hubo 2 o 3 vínculos se disponían paralelamente con pequeña distancia entre cada uno de ellos.

Una gran parte de los vínculos largos se presentaron finos. Por limitaciones del paquímetro en la medición, se calculó el promedio sólo con vínculos mayores a $0,2 \mathrm{~mm}$.

Los resultados biométricos de los vínculos cortos y largos son presentados, respectivamente, en las Tablas II y III.

Tabla II. Registros métricos de longitud y ancho de los vínculos cortos de los Mm. Flexores superficial (MFSD) y profundo (MFPD) de los dedos (Promedios y DE, valores en milímetros).

\begin{tabular}{lcccc}
\hline & \multicolumn{2}{c}{ MFSD } & \multicolumn{2}{c}{ MFPD } \\
\cline { 2 - 5 } Dedos & Longitud & Ancho & Longitud & Ancho \\
\hline Indicador & $6,77 \pm 1,3$ & $8,03 \pm 2,5$ & $6,04 \pm 1,0$ & $9,24 \pm 2,3$ \\
Medio & $6,93 \pm 1,3$ & $7,75 \pm 2,7$ & $6,65 \pm 1,3$ & $10,2 \pm 3,0$ \\
Anular & $7,13 \pm 1,4$ & $8,58 \pm 3,2$ & $6,27 \pm 1,3$ & $10,65 \pm 3,1$ \\
Mínimo & $6,4 \pm 1,3$ & $8,76 \pm 3,2$ & $5,82 \pm 1,3$ & $8,61 \pm 2,4$ \\
\hline
\end{tabular}

Tabla III. Registros métricos de longitud y ancho de los vínculos largos de los Mm. Flexores superficial (MFSD) y profundo (MFPD) de los dedos (Promedios y DE, valores en milímetros).

*= solo un caso.

\begin{tabular}{lcccc}
\hline & \multicolumn{2}{c}{ MFSD } & \multicolumn{2}{c}{ MFPD } \\
\cline { 2 - 5 } Dedos & Longitud & Ancho & Longitud & Ancho \\
\hline Indicador & $10,78 \pm 3,3$ & $1,51 \pm 0,6$ & $7,04 \pm 1,4$ & $1,57 \pm 0,8$ \\
Medio & $12,44 \pm 2,7$ & $1,55 \pm 0,7$ & $8,24 \pm 1,8$ & $1,45 \pm 0,7$ \\
Anular & $9,58 \pm 3,5$ & $1,17 \pm 0,02$ & $7,23 \pm 1,2$ & $1,46 \pm 0,7$ \\
Mínimo & $7,91 \pm 2,4$ & $0,8^{*}$ & $6,7 \pm 1,7$ & $1,52 \pm 0,8$ \\
\hline
\end{tabular}

\section{DISCUSIÓN}

La presencia de los vínculos cortos en todas las muestras observadas en este estudio y siempre en número de uno por cada tendón de los músculos flexores superficial y profundo de los dedos es concordante con autores como Leffert et al. (1974), Ochiai et al. (1979), Woodburne (1984), Hoppenfeld et al. (1994), Yalin et al. (1994), Shao et al. y Moore \& Dalley (2010). En cuanto a la inserción observada en esta investigación, que se extendía desde la cara posterior del tendón hasta la vaina fibrosa en el tercio distal de la falange proximal, ésta también fue descrita por Testut \& Latarjet (1949), Romanes (1972), Ochiai et al. y Woodburne, siendo diferente a lo expresado por Lundborg et al. (1977), Hoppenfeld \& de Boer y Moore \& Dalley, que muestran su localización próximo a la inserción del tendón del músculo flexor profundo en la falange distal.

Los vínculos largos presentan variaciones de presencia, número y morfológicas. En el presente estudio, se observó en promedio en $61,3 \%$ en el músculo flexor superficial de los dedos y en 77,9\% en el flexor profundo. En la literatura consultada, se aprecia valores de frecuencia más elevados con presencia media de $71,9 \%$ en el flexor superficial y de 91,1\% en el flexor profundo (Ochiai et al.; Yalin et al.; Shao et al.). En relación al número de vínculos por tendón, la variación es aún mayor, no siendo posible determinar un patrón, ya que se encontró la presencia de uno, dos, tres vínculos así como también su ausencia, idependiente de estar localizados en los tendones del músculo flexor superficial o profundo de los dedos. Este hecho fue relatado también por autores como Nichols et al. (1954), Leffert et al., Ochiai et al., Zbrodowsky et al. (1981), Woodburne, Hoppenfeld \& de Boer y Shao et al.

En cuanto a la localización de los vínculos largos, en los tendones del músculo flexor superficial de los dedos, se encuentran entre la parte posterior del tendón y la cara anterior de la falange proximal, insertos en la vaina fibrosa, con trayecto oblicuo y próximo a la articulación interfalángica proximal, tal cual fuera descrito por Testut \& Latarjet, Romanes, Zbrodowsky et al., Moore \& Dalley. En cambio, los vínculos del músculo flexor profundo de los dedos están localizados en su mayoría $(86,7 \%)$ entre los tendones de los músculos flexores, superficial y profundo, a nivel de la articulación interfalángica proximal, presentando un trayecto rectilíneo entre esos dos tendones. En 13,3\% de nuestra serie, se localizaron bien próximos a la abertura del tendón del músculo flexor superficial, punto de pasaje del tendón del músculo flexor profundo, disposición también descrita por Hoppenfeld \& de Boer.

El interés en el estudio de los vínculos tendinosos de los músculos flexores se da por su gran participación en la mantención del funcionamiento normal de estos tendones en situación de trabajo y actividades cotidianas, con una contribución vascular y mecánica. 
Autores como Manske \& Lesker (1982) y Shao et al., han considerado a los vínculos como estructuras muy importantes en la irrigación sanguínea de los tendones de estos músculos flexores, observándose la presencia constante de vasos en secciones transversales de estos vínculos, los que son escenciales para garantizar la microcirculación de segmentos intravaginales de los tendones, tanto en situación de trabajo o en el transcurso de la edad. Armentha \& Lehman (1980) complementan, afirmando que los vínculos cortos han sido los principales portadores de irrigación para los tendones superficiales, mientras que los vínculos largos son la fuente principal de irrigación para los tendones profundos.

En lo que se refiere a la contribución mecánica de los vínculos en los movimientos de los dedos, principalmente en presencia de lesión, Stewart et al. han demostrado que el vínculo corto puede facilitar la flexión de los dedos después de una transección distal del tendón, permitiendo a los tendones actuar indirectamente a través de las articulaciones interfalángicas. También afirman que el vínculo corto intacto puede facilitar una gama casi normal de movimientos, a través de las articulaciones interfalángicas, con un $93 \%$ de eficiencia en la flexión de la falange proximal después de lesión del músculo flexor superficial y de $69 \%$ de movimientos normales en la flexión de la falange distal en la lesión del músculo flexor profundo de los dedos. En el mismo sentido, Sasaki \& Nomura (1987) describieron dos casos con flexión completa de la articulación interfalángica proximal, por la presencia de un vínculo corto intacto después de laceración completa de ambos tendones flexores de los dedos, mientras que Flindall \& McGrouther (1991) estudiaron la acción de los vínculos después de dilaceración del músculo flexor largo del pulgar a nivel de la articulación interfalángica y relataron que un vínculo corto del tendón del músculo flexor profundo de los dedos fue encontrado aplicando tracción que producíaflexión de la articulación interfalángica distal.

El conocimiento de la morfología, localización y biometría de los vínculos largos y cortos es de importancia para los cirujanos de mano, involucrados en la reparación y reconstrucción de lesiones que afectan a los tendones flexores, así como también para los fisioterapeutas y terapeutas ocupacionales responsables por el proceso de rehabilitación de las disfunciones de la mano humana.

PEREIRA, E.; GARBELOTTI, S. A. ; PEREIRA, V. R.; MONTIBELLER, R. R.; WAFAE, N. \& OLAVE, E. Anatomic and biometric study of the vincula of the tendons of flexor digitorum superficialis and profundus muscles of the human hand. Int. J. Morphol., 34(2):679-683, 2016.

SUMMARY: The vincula are specialized structures inherent in the flexor tendons of the fingers, and are of great importance in their nutrition. In addition they facilitate flexion of the fingers following a distal transection of this tendon, allowing it to operate indirectly across the interphalangeal joints. The aim of the present study was to describe anatomic and biometric aspects of the long and short vincula of the flexor muscles of the fingers. The work was carried out in hands of 60 adult Brazilian corpses, of both sexes, with an average age between $17.8 \pm 53.1$ years. The short vincula were found in 100 $\%$ of cases of flexor digitorum superficialis tendons and flexor digitorum profundus muscles, and only one in each tendon, with its location always at the extremity of the tendon, prior to insertion in the proximal interphalangeal joint. The long vincula presented great morphological variation, with an average presence of $61.3 \%$ in tendons of the flexor digitorum superficialis and $77.9 \%$ in the flexor digitorum profundus muscle. Furthermore, the number of vincula per tendon changed, and it was not possible to identify a pattern independently of the muscle. With respect to the location of the long vincula, in the flexor superficial tendons they are inserted in the fibrous sheath of the proximal phalanx, with an oblique course and next to the proximal interphalangeal joint. The vincula of the tendons of the flexor digitorum profundus were located (86.7 $\%$ ) between both tendons to level of the proximal interphalangeal joint, presenting a rectilinear course between these two tendons, whereas in $13.3 \%$ they were located very close to the opening of the flexor superficialis muscle tendon for passage of the flexor digitorum profundus muscle tendon. The vincula are essential structures in nutrition and finger motion even when injuries are present. It is therefore, important to have a clear understanding of these structures forproper clinical evaluation in the repair, reconstruction and rehabilitation of hand dysfunctions.

KEY WORDS: Hand; Flexor muscles of the fingers; Long and short vincula. 
PEREIRA, E.; GARbELOTTI, S. A.; PEREIRA, V. R.; MONTIBELLER, R. R.; WAFAE, N. \& OLAVE, E. Estudio morfológico y biométrico de los vínculos de los tendones de los músculos flexores superficial y profundo de los dedos de la mano humana. Int. J. Morphol., 34(2):679-683, 2016.

\section{REFERENCIAS BIBLIOGRÁFICAS}

Amadio, P. C.; Hunter, J. M.; Jaeger, S. H.; Wehbe, M. A. \& Schneider, L. H. The effect of vincular injury on the results of flexor tendon surgery in zone 2. J. Hand Surg. Am., 10(5):626$32,1985$.

Armenta, E. \& Lehrman, A. The vincula to the flexor tendons of the hand. J. Hand Surg., 5(2):127-34, 1980.

Flindall, E. \& McGrouther, D. A. Accessory roles of the vinculum breve of the flexor digitorum profundus and check-rein ligaments at the distal interphalangeal joint. J. Hand Surg. Br., 16(3):305-10, 1991.

Hoppenfeld, S.; DeBoer, P. \& Hutton, R. Surgical Exposures in Orthopaedics. The Anatomic Approach. 2nd ed. Philadelphia, Lippincott, 1994.

Leffert, R. D.; Weiss, C. \& Athanasoulis, C. A. The vincula; with particular reference to their vessels and nerves. J. Bone Joint Surg. Am., 56(6):1191-8, 1974.

Lundborg, G.; Myrhage, R. \& Rydevik, B. The vascularization of human flexor tendons within the digital synovial sheath region-structureal and functional aspects. J. Hand Surg. Am., 2(6):417-27, 1977.

Moore, K. L. \& Dalley, A. D. Anatomia Orientada para a Clínica. $5^{\text {a }}$ ed. Rio de Janeiro, Guanabara Koogan, 2010.

Manske, P. R. \& Lesker, P. A. Nutrient pathways of flexor tendons in primates. J. Hand Surg. Am., 7(5):436-44, 1982.

Nichols, H. M.; Lehman, W. L. \& Meek, E. C. Alteration of the blood supply of flexor tendons following injury. Am. J. Surg., 87(3):379-83, 1954.

Ochiai, N.; Matsui, T.; Miyaji, N.; Merklin, R. J. \& Hunter, J. M. Vascular anatomy of flexor tendons. I. Vincular system and blood supply of the profundus tendon in the digital sheath. $J$. Hand Surg. Am., 4(4):321-30, 1979.

Romanes, G. J. Cunningham's Textbook of Anatomy. 11th ed. London, Oxford University Press, 1972.

Sasaki, Y. \& Nomura, S. An unusual role of the vinculum after complete laceration of the flexor tendons. J. Hand Surg. Br., 12(1):105-8, 1987.

Shao, F.; Tonosaki, A. \& Watanabe, Y. Vincula tendinum of human hands with special reference to vascular patency. Kaibogaku Zasshi, 70(6):569-76, 1995.

Stewart, D. A.; Smitham, P. J.; Gianoutsos, M. P. \& Walsh, W. R. Biomechanical influence of the vincula tendinum on digital motion after isolated flexor tendon injury: a cadaveric study. J. Hand Surg. Am., 32(8):1190-4, 2007.
Testut, L. \& Latarjet, A. Tratado de Anatomia Humana. Barcelona, Salvat, 1949.

von Schroeder, H. P. \& Botte, M. J. Functional anatomy of the extensor tendons of the digits. Hand Clin., 13(1):51-62, 1997.

Woodburne, R. T. Anatomia Humana. $6^{\mathrm{a}}$ ed. Rio de Janeiro, Guanabara Koogan, 1984.

Yalin, A.; Çavdar, S. \& Ercan, S. Vascularization of the long flexor tendon. Okajimas Folia Anat., 70(6):285-93, 1994.

Zbrodowski, A.; Gajisin, S. \& Grodecki, J. Mesotendons of digital flexor muscles and their vasculature. Hand, 13(3):231-8, 1981.

\author{
Dirección para Correspondencia: \\ Silvio Antonio Garbelotti Junior \\ Rua Amadeu Vezzaro, 130, apto $81^{a}$ \\ Bairro Mauá - São Caetano do Sul \\ CEP 09580-710 \\ São Paulo \\ BRASIL
}

Email: silviogarbelotti@hotmail.com

Recibido : 11-01-2016

Aceptado: 11-04-2016 